\title{
Improving out-of-hours intravenous fluid prescribing for junior doctors: a prescription label
}

Sidonie Hartridge-Lambert, Lorna Moore, Oliver Walker, Dominic Wilkinson

\begin{abstract}
Junior doctors are routinely asked to prescribe intravenous fluids (IVF) out-of-hours. Given time constraints and the number of unfamiliar patients, there is uncertainty about their prescription accuracy and safety, particularly in patients who have specific fluid balance requirements. An IVF prescription label was devised for the fluids section of the adult drug chart indicating important patient identifiers and diagnoses such as chronic heart/renal failure. The audit was carried out over a 16 day period, covering three weekends. A qualitative pre- and post-audit questionnaire assessed the confidence of junior doctors in out-of-hours IVF prescribing. All doctors based on the ward during that specific time period were targeted for the post-audit questionnaire. Post-audit, $58 \%(n=7)$ saw the label. $86 \%$ of those seven doctors stated the label had prompted them to do the following: a) examine the patient b) check blood results and c) urine output. $100 \%$ stated that the label improved their confidence in prescribing IVF. In addition, $71 \%$ felt the label made them more cautious in prescribing IVF and $43 \%$ felt they prescribed less. Overall, all seven (100\%) doctors stated that the IVF prescription label was a useful addition to the drug chart. In conclusion, pressurised junior doctors would feel more confident prescribing IVF for unfamiliar patients if crucial information was readily visible on the drug chart. This would improve the accuracy of fluid prescribing and patient safety.
\end{abstract}

\section{Problem}

Junior doctors are routinely asked to prescribe intravenous fluids (IVF) out-of-hours. Given time constraints, it is sometimes difficult to properly check clinical parameters before prescribing. In addition, patients may not be known to the doctor covering on-call duties. Consequently, there is uncertainty about the accuracy and safety of out-of-hours IVF prescribing, particularly in patients who may have specific fluid balance requirements. We wanted to implement a safety intervention that would not only provide basic information relevant to the patient but also improve out-of-hours IVF prescribing accuracy and the confidence of the junior doctor.

\section{Background}

Maintaining an optimal fluid balance in acutely unwell patients, particularly those with co-morbidities, can be difficult within a busy hospital environment. Managing insensible and sensible losses in patients with chronic heart and/or renal failure is a distinct challenge, as is completing an accurate fluid balance chart for patients who are nil by mouth, diuresing, non-catheterised or cognitively impaired.

For many junior doctors starting on the wards, teaching on fluid balance and the correct fluid, volume and flow rate to prescribe is a distant memory from medical school. A recent study evaluating the IVF prescribing practices of Foundation Year 1 (FY1) doctors out-ofhours found significant variability. Although $82 \%(n=149)$ had received teaching on IVF prescribing at medical school, only $48 \%$ had received teaching at their FY1 induction. Given IVF prescribing is a common task for the FY1 doctor, this is unfortunate. Less than half of those surveyed were able to check the medical notes or perform a clinical examination before prescribing. More than half were able to check the patientânds blood results and read their fluid balance and observation charts. The authors recommended this variability be addressed by medical and foundation schools and NHS trusts to improve patient care.

Consequently, junior doctors experience a degree of anxiety and a loss of prescribing confidence on-call when asked to prescribe multiple bags of IVF for unfamiliar patients under significant time pressures. This can result in prescribing variability and potential inaccuracies.

\section{Baseline Measurement}

For this project, we utilised a qualitative questionnaire to establish a pre-intervention baseline. FY1 doctors filled in the questionnaire to evaluate their confidence in out-of-hours IVF prescribing and whether they felt an IVF prescription label would be useful. 85\% $(\mathrm{n}=27)$ stated they had been asked to prescribe IVF for unfamiliar patients (the remaining 15\% had been working in ITU) and $77 \%$ felt that the label would be a useful prescribing aid. Importantly, 100\% of all FY1s surveyed said they would be willing to trial the label on their ward.

See supplementary file: Example of a DAPS sticker on drug chart_0.png

\section{Design}

Having received a positive response from the FY1 doctors regarding the concept of an IVF prescription label, we devised a simple label for the fluids section of the adult drug chart. The label 
progressed through several designs until we reached an informational and aesthetic conclusion. It was important the label remained simple and easy to complete. The final design was then reviewed and approved by the hospital pharmacy. Quick to use, it functioned as a prompt for the junior doctor. It detailed basic patient identifiers and detailed 'yes/no' questions relating to chronic heart/renal failure. Other important factors relating to fluid balance could be noted in a free text section entitled 'other'. The label was to be filled in by the patient's primary medical team and signed and dated. The label's size and expiration date allowed a degree of flexibility as several labels could be applied across the drug chart to reflect changes in fluid balance (for example, a patient admitted with acute kidney injury whose blood parameters improved). It was not our intention to replace the need for careful clinical assessment of all patients when prescribing IVF but to highlight those who had specific fluid balance requirements to the busy on-call junior doctor.

The audit ran for a 16 day period (covering three weekends) on an acute medical ward. Permission was gained from senior clinical personnel. A publicity campaign ("are you up for a fluid challenge?") ran prior to the launch with posters placed in key places around the hospital and via word-of-mouth, particularly to those doctors who would be on-call during the audit period.

\section{Strategy}

The first label was initially trialled on a busy elderly care ward. Unfortunately, as the label utilised light grey text on a plain white background, the medical teams did not see it on the drug chart and did not fill it in. We therefore acknowledged that there were difficulties with the aesthetic and focussed on a re-design. This involved the use of colour (a red border) and the addition of the word 'caution' at the top, centred, highlighted with fluorescent yellow to draw attention. We also included the 'Doctors Advancing Patient Safety' (DAPS) logo.

The label was then re-trialled on a second, acute medical, ward with a resumption of our advertising campaign featuring the new design. We received positive feedback on the design from all medical personnel (including nursing staff) and compliance was excellent, with all labels completed appropriately.

\section{See supplementary file: Example of DAPS stickers.png}

\section{Post-Measurement}

All doctors based on the ward or on-call for medicine during that specific time period were targeted for the post-audit questionnaire. Simple analysis was carried out in Microsoft Excel. Post-audit, 58\% $(n=7)$ saw the label. Out of those seven, $86 \%$ stated the label had prompted them to do the following: a) examine the patient b) check their blood results and c) check urine output. All seven (100\%) stated that the label improved their confidence in prescribing IVF. In addition, $71 \%$ felt the label made them more cautious in prescribing IVF and $43 \%$ felt they prescribed less IVF. Overall, all seven doctors (100\%) stated that the IVF prescription label was a useful addition to the drug chart.

\section{Lessons and Limitations}

We learnt a few lessons during the completion of this project:

1. Clinical staff are already overburdened with paperwork therefore it was important that the label remained simple and easy to use with a perceived and actual benefit to enhance compliance. Involving the ward clerk and ward pharmacist was useful to assist with the distribution and application of labels on all the charts.

2. Aesthetics were important to ensure the label was noticed on the adult drug chart. A good advertising campaign was also vital to raise awareness and successfully launch the project.

3. Out-of-hours IVF prescribing is not simply a concern for junior doctors but for all staff, as demonstrated by positive, anecdotal feedback we received from nurses, senior house officers and specialist registrars. Therefore, the label will be useful for all healthcare professionals to improve patient care and safety.

The main limitation of the project was the small sample size: We were reliant on a small number of doctors being called to the ward out-of-hours. In addition, due to an increased focus on appropriate IVF prescribing by the nursing and medical staff, the primary medical teams were also ensuring that appropriate fluids were prescribed before the end of their shift, therefore obviating the need for nurses to bleep the on-call doctor.

\section{Conclusion}

Pressurised junior doctors would feel more confident prescribing IVF for unfamiliar patients if crucial information was readily visible on the drug chart. We feel that our label improved the accuracy of fluid prescribing, prescribing confidence and overall patient safety. Further studies are required to evaluate whether this translates to improved outcomes. In order to facilitate this, we have submitted a business case to the trust to implement the intervention on a wider scale and complete a larger audit.

\section{References}

1) Lim CT, Dunlop M, Lim CS. Intravenous fluid prescribing practices by foundation year one doctors - a questionnaire study. Journal of the Royal Society of Medicine Short Reports. 2012;(3):64. 